\title{
The Impacts of Biotechnology on Biodiversity in Global Health: A Case Study on Avian Influenza in Bangladesh
}

\author{
Muhiuddin Haider ${ }^{1}$, Milind Patel ${ }^{2}$, Priyanka Bhattacharje ${ }^{3}$, Maariya Bassa ${ }^{4}$ \\ ${ }^{1}$ School of Public Health, University of Maryland, USA \\ ${ }^{2}$ College of Agricultural \& Biological Engineering, Purdue University, USA \\ ${ }^{3}$ Department of Genetic Engineering \& Biotechnology, Shahjalal University of Science \& Technology, Bangladesh \\ ${ }^{4}$ School of Nursing, Yale University, USA
}

\begin{abstract}
Article Info
Article history:

Received Feb 18, 2015

Revised March 23, 2015

Accepted Apr 26, 2015

Keyword:

Avian

Bangladesh

Biodiversity

Biotechnology

Influenza

ABSTRACT

Biodiversity is the variability of between genetics, species, or ecosystems of living organisms within a specific region. Biodiversity is essential for sustaining healthy living networks and systems because it allows for a variety of food sources, medicine, and biological control, while also playing a significant role in atmospheric regulation, nutrient cycling, and pollination. Loss of biodiversity and ecosystem change increases the risk of the emergence or spreading of infectious diseases and global pandemics such as the Avian Influenza (AI H5N1). Biotechnology is one solution for reducing, and ultimately eliminating, the transmission of avian influenza. Traditional methods of treating infected animals, such as common vaccines, are temporary solutions that have no effect on the biodiversity of an ecosystem. Methods in animal biotechnology such as artificial insemination, embryo transfer, and in vitro fertilization have led to developments of cheaper, safer, and more effective vaccines. Livestock that have been treated for H5N1, as well as those that are healthy and have never been infected have proven to increase the diversity, leading to the elimination of specific issues. Similar effects are attainable if these animal biotechnology methods were to be used on poultry infected with the avian influenza virus.
\end{abstract}

Copyright () 2015 Institute of Advanced Engineering and Science. All rights reserved.

\section{Corresponding Author:}

Milind Patel,

College of Agricultural \& Biological Engineering,

Purdue University,

225 S. University Street, West Lafayette, IN, 47907, USA.

Email: patel231@purdue.edu

\section{INTRODUCTION}

Biodiversity is the variability of between genetics, species, or ecosystems of living organisms within a specific region. Biodiversity is essential for sustaining healthy living networks and systems because it allows for a variety of food sources, medicine, and biological control, while also playing a significant role in atmospheric regulation, nutrient cycling, and pollination [1]. Loss of biodiversity and ecosystem change increases the risk of the emergence or spreading of infectious diseases and global pandemics such as the Avian Influenza (AI H5N1).

Many Asian countries, such as Bangladesh, are chronically infected with AI. In recent years, highly pathogenic avian influenza (HPAI H5N1) has caused massive mortality in domestic poultry flocks, infected birds in $41 \%$ of the avian orders, and has led to 2 human deaths. The root causes for the emergence, persistence, and spread of H5N1 includes: Changes in pathogen and host biodiversity; Disappearance and degradation of wetlands; Deterioration of landscape along with bird migrating routes; and cultural practices of poultry production and marketing. Biotechnology's role in increasing biodiversity can limit the emergence, persistence, and spread of AI. 
An analysis of biodiversity's role in reducing the burden of disease from various articles and journals will be discussed. Also, the various options and potential of biotechnology and its relationship in biodiversity will be analyzed. After analyzing the data and multitude of options, a final discussion about biotechnology's role in reducing avian influenza in Bangladesh will take place. A strong conclusion about biotechnology's role in biodiversity with the special focus on the Avian Influenza in Bangladesh will be developed. The objectives are to find: What is the relationship between biotechnology and biodiversity? How biotechnology is useful in reducing the burden of disease? What needs to be done in order to promote biotechnology's role in reducing disease transmission through research?

\section{LITERATURE REVIEW}

Biodiversity's role in reducing the burden of disease from various articles and journals will be discussed along with the various options and potential of biotechnology and its relationship in biodiversity.

\subsection{Global Patterns of Avian Influenza in Wild Birds}

Avian influenza viruses have been seen in many different species, but it is thought that wildfowl and shorebirds are virus reservoirs in nature. The highly pathogenic avian influenza (HPAI) virus phenotype is common in wild birds and poultry, and it is achieved by introducing an amino acid into the bird [2].

\subsubsection{Migratory Birds as a Natural Reservoir of LPAI Viruses}

Birds of wetlands and aquatic environments establish the major natural LPAI virus reservoir. The LPAI viruses infect cells that line lining the intestinal tract of birds, and the viruses are excreted in high concentrations in their feces. Finding a high amount of viruses in birds that live in aquatic environments may be due to the virus being transmitted from the fecal matter to the birds via surface waters. See Table 1 in Olsen et. al., 2006, which displays wild birds in which the influenza A virus is prevalent [2].

Many Anseriformes and Charadriiformes perform regular long-distance migrations to different countries and continents; therefore they potentially distribute LPAI viruses to different parts of the world. Whether it is on land or along the major flyways (the routes that birds fly during migration), migration connects many bird populations through common breeding areas, during migration, or at shared nonbreeding areas. As a result of the migrations and interactions between infected birds and other species, the pathogen is transmitted and the virus spreads to new areas [2].

It is also important to note that while birds migrate from breeding to non-breeding areas, they make several stops in between to eat, rest, and prepare for their next flight. The birds spend more time during these stops than they do during their actual flight. These stops result in high local-densities of infected birds, and the sites are important for the transmission of LPAI viruses between wild and captive birds, as well as other species in the area. Maps of migration patterns for wild birds can be seen in Figure 1 of Olsen et. al, 2006 [2].

\subsubsection{Influenza Viruses in Ducks}

H5N1 spreads among wild ducks in North America and Northern Europe. Dabbling ducks (Anas genus) and Mallards (Anasplatyrhynchos) are infected with influenza viruses more frequently than other bird. This can be related to dabbling ducks using food found at surface waters as their primary consumption and switching breeding grounds between years [2].

This information is known or observed in ducks in the Northern Hemisphere, but there is not much known about the prevalence of avian influenza in wild ducks in the Southern Hemisphere, or the potential of transmission between the two hemispheres [2].

The most feasible method to prevent and control H5N1 HPAI in wild birds is through control of the disease in poultry with use of vaccine to reduce environmental burden of H5N1 HPAIV, and eventual eradication of the virus in domestic poultry, especially in domestic ducks which are raised in enzootic countries on range or in other outdoor systems having contact with wild aquatic and periurban terrestrial birds [3].

\subsubsection{Influenza Viruses in Gulls and Terns}

Late summer and early fall is the time period in which the virus is at the highest prevalence in gulls, even though influenza viruses are detected in only a small proportion of gulls. Also, gulls breed in crowded and highly dense colonies making it easier for the virus to spread, which is different from dabbling ducks which are more spread out in their breeding colonies [2]. 


\subsubsection{Influenza Viruses in Waders}

Waders contain a wide variety of viruses. Unlike ducks, waders have higher virus prevalence during the spring migration $(\sim 14 \%)$ than any other time of the year. Many wader species of the Northern Hemisphere Many wader species of the Northern Hemisphere migrate internationally over long distances; therefore it is very likely that they spread the influenza virus all over the globe [2].

\subsubsection{Influenza Viruses in Other Wild Birds}

Numerous birds host LPAI viruses but it is unclear which species contain an endemic for influenza viruses and which species contain the virus as a temporary pathogen. Geese, swans, rails, petrels, and cormorants are species in which influenza viruses are frequently detected. These species share a habitat with species in which influenza viruses are endemic or indigenous. Influenza virus prevalence seems to be lower in these birds than dabbling ducks, but studies are limited and have not been carried out during certain seasons and in certain locations [2].

Behavior and ecology are important for determining the role of ducks, gulls, and waders as host species. Geese are mainly herbivorous and usually gather in large flocks for grazing in pastures and agricultural fields, especially during the nonbreeding season [2].

In parts of the world, wild geese and ducks are frequently kept alongside chickens, which may form the bridge for influenza viruses between wild and domestic birds [2].

\subsubsection{Genetic Variation of Influenza Viruses in Wild Birds}

During the winter, the majority of tundra shorebirds from the Russian Far East spend the season in Southeast Asia and Australia. The ecological isolation of influenza virus hosts allows occasional spillover of gene segments from one gene pool to the other, and genetic reassortment occurs due to the segmented nature of the influenza virus genome [2].

\subsubsection{H5N1 in Wild Birds}

It is strongly believed that domestic waterfowl, specific farming practices, and agroecological environments played a key role in the occurrence, maintenance, and spread of H5N1 for many affected countries. There has been debate over whether infected birds cause the virus to spread (as mentioned throughout the first several paragraphs) because it has been argued that infected birds become severely affected and cannot continue migration, thus making it unlikely to spread the H5N1 virus [2].

On the contrary, it has been seen in experimental infections that several bird species survive infection and shed the H5N1 virus without apparent disease signs. Also, it has been seen in many wild birds and chickens that birds become partially immune after previous exposures to LPAI influenza viruses. Recent studies suggest that HPAI viruses may become less pathogenic to ducks infected experimentally, while retaining high pathogenicity for chickens [2].

Now that we have an understanding of AI in birds, we will discuss the nature of avian influenza viruses in wild birds and migration patterns in the next section.

\subsection{Avian Influenza Viruses in Migrating Wild Birds \\ 2.2.1. Inter-Species Transmission}

The ecological relationships between wild birds and avian influenza have resulted in a broad pool of viral genetic diversity as well as a reservoir of viruses that could be transmitted among species. Understanding how these relationships work can better help us understand how to reduce the transmission of avian influenza into domestic birds and humans [4].

Wild aquatic birds such as ducks and geese are a reservoir for avian influenza viruses, and the majority of avian influenza viruses detected in wild birds are low pathogenic (LPAI). Poultry are a key link between wild birds and people when looking at human infections. Transmission of H5N1 from birds to humans has occurred largely with poultry outbreaks, suggesting that the transmission occurs during higher viral amplification and systematic spread. This view is supported by the emergence and spread of H5N1 in southern China [4].

\subsubsection{Intra-Species Transmission}

Human-to-human transmission of avian influenza is extremely rare that it is basically non-existent. Transmission of H5N1 requires contact between humans and infected birds. Avian influenza viruses cause seasonal epidemic influenza where mutation and reassortment within the virus causes new strains to emerge each year in within infected humans. These outbreaks occur every year due to reassortment and mutations of the virus without new viruses or infections being introduced from birds [4]. 


\subsubsection{Viruses in the Cell}

The avian influenza virus is divided into eight segments of single stranded RNA, and the viral surface antigens are important when it comes to virus entry into host cells. Wild birds are considered the natural reservoir for influenza A viruses becauseall of the currently known HA subtypes (H1-H16) have been detected in wild birds, and there have been hundreds of thousands of infections in humans to three HA subtypes (H1-H3) over the past 100 years. Over the past decade, human infections to H5N1, H7N7, and H9N2 have risen with both symptomatic and asymptomatic outcomes. H5N1 was introduced to humans directly from poultry, and other HA subtypes have been introduced to humans through pigs and other species, which were infected by the virus from birds [4].

The virus must interact with receptors (recognized by the virus antigens) in order to enter the host cell. Typically, infection of LPAI viruses occurs following ingestion of virus-contaminated mediums such as feces and contaminated water. On the contrary, HPAI viruses encounter changes in amino acids at the HA cleavage site, which effects the process of systemic replication. According to studies, the H5N1 viruses that have emerged in Asia replicate more efficiently in the trachea of ducks rather than the intestines, and these studies are important in helping us understand how the virus spreads amongst wild bird populations such as inhalation vs. fecal [4].

Once a viral strain gets inside a host cell, the possibility of rapid viral evolution due to mutation is present, and RNA viruses have the highest chance of mutating due to the lack of proofreading ability during replication. These mutations can result in multiple strains of genetically diverse viruses being isolated from an individual who might have been originally infected with a single strain. Also, a new hybrid virus can form if the host cell is infected with two distinct viral strains because reassortment can occur between the gene segments. These mutations involving reassortment occur in birds, humans, and other species [4]. An example of a mutation is the concern of H5N1 from a bird infected a person that is already infected with H3N2 (which causes the seasonal flu) resulting in highly transmitted viruses, which has the potential of becoming highly pandemic [4].

\subsubsection{Viruses on the Wing}

Wild birds will travel short and long distances to seek out habitats that are favorable for survival and reproduction. Any movement from one area to another can result in the bird acquiring avian influenza viruses. For example, genetic similarities between HPAI H5N1 viruses obtained from migrating ducks at Poyang and Qinghai Lakes in China, which are separated by 1700 km, provide strong evidence that migrating birds can carry AI viruses over long distances. These investigators concluded that the ducks sampled at Poyang Lake were most likely infected with viruses from local poultry while over-wintering in southern China [4].

It is clearly seen that viruses travel along with birds as they migrate. With this information known, what becomes of the viruses as they travel is an important question. When a virus is detected in migrating birds, it does not mean that the virus will be introduced into the new geographic area in which the bird is migrating to. A time period of 3 weeks is when exposure to the break of virus shedding is likely to occur. Therefore, detection of viruses in birds that have been resident in a given area for over 3 weeks is likely to indicate transmission is occurring in that area [4].

In Asia, it seems that infected poultry serve as a reservoir with spillover into the wild bird population. This can be seen from intensive sampling from the past few years where relatively few wild birds had been infected, and most of the HPAI H5N1 isolates came from sick and dead wild birds [4].

\subsubsection{Viruses in Populations and Environment}

In North America, the prevalence of avian influenza viruses appears to be highest at northern breeding grounds when large numbers of different-aged birds, originating from many different locations, gather and raise young. As the bird migrates south, prevalence decreases because cool and wet conditions (compared to other locations a bird might travel) favor virus transmission [4].

In 2005 at Qinghai Lake, China, over 6000 geese, gulls, cormorants, swans and ducks died at the lake during May and June. This outbreak was not caused by a single strain of H5N1; instead, sequence analysis of 15 viruses from 6 species of wild birds showed that at least 4 genotypes of H5N1 were associated with the outbreak. This finding adds to the growing evidence that avian influenza viruses may not be genetically stable in wild aquatic birds [4].

Poultry are usually raised under conditions that heighten opportunities for influenza transmission, which leads to increased opportunities for mutation and reassortment.Older poultry increases the chance of contracting the virus. Also, the market, depending on if it is a live trade market or other factors, can affect contraction of the disease [5]. These conditions exist in ecologic settings where wild birds are concentrated at breeding grounds, increasing the chance of virus transmission between species [4]. 


\subsubsection{The Nidus Concept and Critical Control Points}

The nidus (from the Latin word for nest) of a disease is the location where host, pathogen, and environmental conditions come together to produce a disease (outbreak of a disease). Several resources of evidence have shown that viral strains can be sustained by the movement of poultry and poultry products without the introduction or reintroduction from free-ranging wild birds [4].

The chance of human infection of avian influenza increases at locations where frequency of contact or exposure to infected birds is high. These locations have increased opportunities for virus transmission and evolution. Removing all live birds for a certain amount of time will eliminate existing viruses in the environment [4].

At the end of the breeding season, migration of birds decreases the population of the breeding grounds thus lowering the amount of virus that sheds into the environment. Over several months, the amount of infective virus in the environment decreases and abiotic factors such as the heat, temperature, and solar radiation "disinfect" the environment [4].

Wild birds serve as a reservoir of avian influenza viruses and genes that can infect other animals (dogs, cats, pigs, horses, etc.) and humans. Also, migrating birds can spread viruses to new geographic areas. But the biggest threat to human health comes from locations where humans have altered the ecology of the host, pathogen, or environment. Since humans keep certain animals nearby for food or companionship, it is more likely to pick up the virus from these animals over wild birds [4].

After looking at patterns and trends of AI transmission among birds, we will look at the impacts of biodiversity on the emergence and transmission of infections such as avian influenza.

\subsection{Biodiversity and the Emergence and Transmission of Infectious Diseases}

In June of 2010, the Intergovernmental Science Policy Platform on Biodiversity and Ecosystem Services (IPBES) was established to view how changes in biodiversity affect humans, and it has been noted that humans would be deeply affected if there were a loss in biodiversity. Setbacks to ecosystem functions would be one of several noticeable changes [6].

Biodiversity involves the diversity of genes, species, and ecosystems, but due to the growth of the human population, a loss of biodiversity has occurred. This loss of biodiversity has threatened the extinction of many plant and animal species because the change to Earth's ecosystem and climate affects the demands of food, fresh water, fuel, and other resources [6].

Changes in biodiversity also have the potential to change disease infection amongst plants and animals. Biodiversity plays two roles in the emergence and transmission of infectious diseases. A high biodiversity may result in more pathogens, but on the other hand, biodiversity can reduce pathogen transmission for diseases that have been long established and those that have recently emerged [6].

\subsubsection{Biodiversity and Pathogen Transmission}

Transmission of Pathogens between Species

The loss of biodiversity can affect the transmission of infectious diseases by changing the abundance, behavior, and condition of the host, vector, or parasite. In recent years, it has been seen that the loss of biodiversity usually increases pathogen transmission and disease incidence. Specifically, this correlation occurs in ecological systems that have a variety of pathogens, hosts, ecosystems, and transmission modes. An example of this is the West Nile virus where it has been seen that communities in the United States with low avian diversity are dominated by the species that spread the virus, resulting in a high volume of infected mosquitoes and humans [6].

Another idea has emerged on how the loss of a species can increase the transmission of diseases in another way. This idea is the effect of a higher density of a species in a community, which is compared and contrasted with the commonly known idea of reduced diversity. It has been confirmed from recent experiments that disease transmission can increase when species richness declines, even if the host density stays constant. Although if that species were a host for a pathogen, then the loss of that species would reduce the number of hosts, which means the transmission of disease would decrease, assuming all other factors remain constant. Table 1 of Keesinget. al., 2010, shows several diseases that have increased transmission due to loss of biodiversity [6].

In conclusion, reducing biodiversity can increase disease transmission when the lost species are not hosts for the pathogen. For pathogens for which transmission is a function of host density, loss of diversity is most likely to increase transmission if the loss causes an increase in the diversity of adequate hosts [6].

Species Diversity versus Species Identity

The transmission of disease is related to species "richness" and diversity in a couple of ways. On one hand, if a host species thrives as biodiversity is lost, then disease transmission will increase as 
biodiversity decreases. On the other hand, if biodiversity decreases and the host species begins to die out, then disease transmission will decrease. In several case studies, the species that are most likely to reduce pathogen transmission are most likely to be lost from ecological communities as diversity declines. For example, in a field study in Oregon, USA, the ubiquity of hantavirus Sin Nombre virus rose from $2 \%$ to $14 \%$ as the mammalian species diversity declined. An experimental study in Panamá was conducted where the small-mammal diversity was reduced by trapping and removing species that are not hosts for the virus, and it was seen that the reduced-small mammal diversity caused an increase in the density of the host species, which resulted in an increase of hantavirus transmission [6].

\section{Diversity within Individual Hosts}

Several recent studies have begun to show that changes in the biodiversity of an organism's "microbiome" can alter pathogen transmission. For example, the number of microbes in corals that have white plague disease is different from the number of microbes in healthy coral. A few correlation studies have been conducted, but it has still been difficult to determine whether changes in microbial communities are the cause or consequence of infections. Some experimental studies have cleared this up by showing that an increase in microbial biodiversity can indeed protect against infection. For example, children with a history of ear infections given a mixture of five strains of Streptococcus were less likely to develop subsequent infections compared to a control group. Also, the opposite occurs when microbial biodiversity is decreased, which is disease transmission increases. When mice with consistent infections of $C$. difficile were treated with antibiotics that reduced the intestinal microbe diversity, they began shedding $C$. difficilespores at high rates [6].

Another way that high microbial species diversity can help prevent disease transmission is by preventing the colonization of the invasive pathogenic species. For example, the more diverse the microbiome surrounding the roots of wheat plants, the more protected the plants were against invasion by the pathogenic bacterium Pseudomonas aeruginosa [6].

\subsubsection{Biodiversity and Pathogen Emergence}

There are two hypotheses that relate biodiversity to disease transmission. The Amplification Effect states that biodiversity and disease transmission have a positive correlation, and the Dilution Effect states that biodiversity and disease transmission have a negative correlation [6].

It is already known that if an ecological community already contains a pathogen or disease, then a loss in biodiversity will increase the rate of transmission [6]. But if a new pathogen emerges, a loss in biodiversity may have a different effect. For example, finding a new host for a pathogen involves multiple steps (such as the initial invasion into the new host, known as "spillover"), each of which a change in biodiversity has a different effect. In a recent analysis, the probability of the emergence of pathogens from wildlife to humans was positively correlated with mammalian wildlife species richness [6].

Once spillover occurs, a high density of the host species may establish a pathogen, and spread transmission among the new host species. For example, the Nipah virus spilled over from wild fruit bats to domestic pigs in Malaysia; high densities of pigs in local farms appear to have spread established pig-to-pig transmission, and the pathogen then spilled over from pigs to humans [6].

After a look and analysis of the impacts of biodiversity on the emergence and transmission of infections such as avian influenza, next we will focus on avian influenza outbreaks in chickens in Bangladesh.

\subsection{Avian Influenza Outbreaks in Chickens in Bangladesh \\ 2.4.1. Main Point}

The investigation showed that the epicenter of the HPAI outbreaks in Bangladesh was the Sarishabariupazila of Jamalpur district, with the primary source of infection being backyard chickens. Analysis of the H5N1 isolates showed "Qinghai lineage," closely related to viruses isolated from Russia, Mongolia, and Afghanistan. Therefore, the virus probably entered Bangladesh through migratory birds [7].

\subsubsection{Sources of Outbreaks}

Bangladesh is specifically vulnerable to virus perpetuation because of insufficient biosecurity, rearing of chickens and ducks together, selling live birds, and deficient disease surveillance [7].

Through July 10, 2007, 52 outbreaks caused by H5N1 were investigated. The chickens were on a farm, and they were examined by an upazila (a lower administrative unit of Bangladesh) veterinarian. Pretested questionnaires administered by 2 veterinarians were used to collect information about the farms with the infected chickens. The veterinarians made observations about the farms and recorded them. They also interviewed the farmers and allied personnel. Through another questionnaire, data was collected on 
commercial and backyard farms and outbreaks from the upazila livestock offices. The data can be seen in Table 1 of Biswaset. al., 2008 [7].

It was hypothesized that the virus came from chickens in nearby farms. On March 26, 2007, outbreaks peaked in 11 affected farms in Dhaka, Gazipur, and Narayangogj. Since a cluster of infections formed, the source (a common source) may have been larger live bird markets. A map tracking the spread of HPAI outbreaks in chickens in Bangladesh can be seen in Figure 2 of Biswaset. al., 2008 [7].

Looking at 9 possible sources, egg trays and contaminated vehicles from larger live bird markets and local live bird markets accounted for $47 \%$ of probable virus sources, eggs for $48 \%$, and healthy chickens for $5 \%$. For backyard chickens, sources of the virus spread were selling chickens (5\%), giving chickens to relatives or neighbors (15\%), moving birds through local poultry vendors, and hiding birds during extracting operations (10\%) [7].

In January of 2008, a child was diagnosed as positive for H5N1 by the US Centers for Disease Control and Prevention, the first case of human infection in Bangladesh. Several sources for the lack of human cases may be early immunological response, genetic variation in receptors, poor surveillance of diseases in humans, or using antiviral drugs while extracting from birds [7].

Now that we have an understanding of AI outbreaks of chickens in Bangladesh, we will finally look at the biodiversity of specific regions in Bangladesh.

\subsection{Biodiversity of Birds of DingaputaHaor and Its Surrounding Area of MohangongUpazila, Netrakona District}

\subsubsection{Main Point}

The quickly growing human population in Bangladesh has made drastic impacts on the wetland biodiversity of the country. Recent developments showing a lack of awareness of the importance of wetlands have resulted in wetlands being continuously lost or degraded [8].

\subsubsection{Introduction}

A few terms to be familiar with when studying the ecosystem of Bangladesh are beel (lake-like wetland), baor (oxbow lake), and haor (wetland ecosystem). Bangladesh contains 150 species of waterfowl and 24 species of mammals [8].

\subsubsection{Materials and Methods}

Data taken from a previous study was taken in the Netrakona District in the Dhaka division of Bangladesh, from July to October of 2010 was analyzed to determine the bird biodiversity and the factors affecting the biodiversity. The biological environment covers a vast area of faunal species, with some waterfowls, birds, and mammals have being documented as faunal species. The data from the study was collected directly by talking to fishermen and farmers, and from secondary sources such as fisheries offices and agriculture offices at MohonganjUpazila. To see a map of area that was analyzed, see Figure 1 of Islam et. al., 2013 [8].

\subsubsection{Results and Discussion}

Deforestation has decreased the biodiversity of the Dingaputahaor. Many of the aquatic plants that were grown in the area were used as food, medicinal plants, duck feed, or fuel. 19 timber plants, 28 fruit plants, 13 ornament plants, and 11 species of medicinal plants have also been found in the area. Along with many plant species, 24 agricultural crops, 18 aquatic weeds, and 39 land weeds were recorded in the area [8].

Every winter, many migratory birds flock to this haorto make a temporary habitat. The common names for the 11 migratory birds are: Fulvous Whistling-Duck, Gadwall, Northern Pintail, Gagany, Common Teal, Red-Crested Pochard, Ferruginous Duck, Tufted Duck, Little Cormorant, and Pallas’s Fish-Eagle. A more detailed list can be seen in Table 9 of Islam et. al., 2013 [8].

There have been 18 waterfowl species recorded, and migratory, resident, and domestic waterfowls were identified in the wetland area. Usually, duck rearing is a good practice for women in the area. The common names for the 18 waterfowl birds are: Greylag Goose, Duck, Kingfisher, Great Egret, Cattle Egret, Grey Heron, Black Heron, Night Heron, Stork, Plover, Hemipode, Water Pipet, Crested Grebe, Diver, White Pelican, Brown Fish Owl, Brahiminy Kite, and Osprey. A detailed list of waterfowl birds can be seen in Table 10 of Islam et. al., 2013 [8].

8 species of bank birds were recorded in the area: Common Myna, Common Starling, Bulbul, Dove Sociable Lapwing, Magpie Robin, Woodpecker, and Weaver. A detailed list of bank birds can be seen in Table 11 of Islam et. al., 2013 [8]. 


\section{RESEARCH EFFORTS THROUGH BIOTECHNOLOGY}

There are 4 main areas of biotechnology that can directly assist vaccine devlopment: Tissue culture (in vitro technologies), molecular (DNA) marker technology, molecular diagnostics, and cryopreservation [9].

\subsection{Current Vaccination Methods}

In a study conducted by Swayne et. al. (2009), 3-week-old chickens (Gallus domesticus) (n = 11), 2week-old domestic ducks (Anasplatyrhynchos) ( $\mathrm{n}=11$ ), 73-week-old reproductively active turkey hens (Meleagrisgallopavo) $(\mathrm{n}=9)$, 3-week-old turkey poults $(\mathrm{n}=11)$, and 5-week-old Japanese quail (Coturnix japonica) $(\mathrm{n}=11)$ were intranasally inoculated with $10^{5}$ mean chicken embryo infectious doses of A/Mexico/4108/2009(H1N1) to determine infectivity potential. Five uninfected chickens, ducks, turkey poults, and quail, as well as three uninfected turkey hens were contact exposed to intranasally inoculated birds to assess transmission potential. The observation lasted 15 days, with tests taken after 2, 4, 7, and 10 days [10].

During the observation period of 15 days, clinical signs did not develop in any of the birds, and none of the birds died. The virus was not highly pathogenic for chickens. No virus was isolated in chicken eggs from swabs or tissues from chickens, turkeys, or ducks. Also, all the chickens and turkeys were negative for antibodies to the virus. One intranasally inoculated duck had a hemagglutination inhibition (HI) antibody [10].

The main point to takeaway from this study is that certain vaccines are still effective in reducing certain diseases (H1N1) in poultry, but only for specific species. Biotechnology on the other hand has more versatility when it comes to how many species it can treat. Also, biotechnology's results are more durable and long-term than vaccines, which may temporarily solve issues, but as seen in the study (conducted in 2009), the effects still linger.

\subsection{Methods Involving Biotechnology}

Livestock account for over half the agricultural output of Bangladesh. Current technology is not efficient enough to keep up with the increasing demand of animal products for the growing population. Biotechnology on the other hand has proven to advance the production and health management of farm animals as evidenced by 11 types of veterinary biologics that have been produced over the years for the treatment of major diseases among livestock and poultry. When looking at fish species, modern biotechnology techniques have been able to produce $46,000 \mathrm{~kg}$ of different fish species, which is significantly greater than the 5,000 kg that are produced via natural spawning.

Animal genetic resources need to be conserved for future generations. The use of artificial reproduction is a very useful tool in the conservation of endangered species. Modern reproductive biotechnologies, such as artificial insemination, embryo transfer, in vitro fertilization, gamete embryo micromanipulation, semen sexing, genome resource banking and somatic cell nuclear transfer (cloning), all have enormous potential for conserving rare breeds of livestock.

Biotechnology can be used to make stronger vaccines that are more effective than the traditional vaccines we have today. Using vaccines containing live attenuated viruses can prevent very few diseases. The risk with these vaccines is the chance that the virus reverts back to a harmful state and causes further infection. Vaccines that contain inactivated viruses are also a risk because the batch of viruses may not be fully killed leaving a few living viruses in the vaccine that can cause infection [11].

Biotechnology offers the development and production more effective, safer, and cheaper viral vaccines. Recombinant DNA technology has made highly purified biologically active proteins possible for production on a large scale. Purified proteins are essential for effective vaccines, and DNA technology has primarily been focused on the production of protein subunits to develop vaccines. Scientists have been using DNA technology over the past few years to find an expression system that will provide the protein modification that is similar to naturally occurring proteins, as well as produce large quantities of desired gene products [11].

\subsubsection{Marker Assisted Selection Technique}

Marker assisted selection technique is the most promising tool of biotechnology. DNA markers are used to increase the response to selection in a population. Molecular markers are segments of plant DNA that breeders use to detect the presence or absence of specific alleles in experimental plants, thus using them as selection tools. Several marker systems, such as Restriction Fragment Length Polymorphisms (RFLPs) and Random Amplification of Polymorphic DNAs (RAPDs), have been developed and applied to a range of crop species with higher efficiency [12]. 


\subsubsection{Artificial Insemination (AI)}

Artificial insemination is the introduction of semen into the vagina or cervix of a female by any method other than sexual intercourse [13]. This method is used for the purpose of reproduction, and its main goal is to quickly and efficiently pass on desirable characteristics of a bull or other male livestock animal. This technique is useful in livestock farming. AI can be used to improve reproductive efficiency in farm animals. The greatest advantage of artificial insemination is the opportunity to spread superior germplasm by the wide use of carefully tested and selected sites.

\subsubsection{Embryo Transfer (ET)}

In ET, a donor cow of superior breeding is chemically induced to super ovulate, and the fertilized eggs are then collected from the donor and are subsequently transferred to recipient females, which serve as surrogate mothers for the remainder of pregnancy. Embryo transfer techniques allow top quality female livestock to have a greater influence on the genetic advancement of a herd or flock, and it has been applied to nearly every species of domestic animal as well as many species of wildlife and exotic animals [8]. The general epidemiological aspects of embryo transfer indicate that the transfer of embryos provides the opportunity to introduce genetic material into populations of livestock while greatly reducing the risk for transmission of infectious diseases.

\subsubsection{In Vitro Fertilization}

In vitro fertilization (IVF) is a process by which egg cells are fertilized by sperm outside the womb, and it is used widely to ensure a higher rate of fertilization and select desirable genetic traits. During IVF, unfertilized eggs (oocytes) removed from the donor cow's ovaries (usually recovering 6-8 useable oocytes) mature in an incubator and are fertilized with sperm. Fertilized eggs are cultured and allowed to develop in vitro for a few days. Embryos at the 8-cell stage (blastocyst stage) are then transferred into a female recipient, where implantation and embryo development can occur [14].

\subsection{Application of Biotechnology in the Conservation of Biodiversity}

Biodiversity is the source for crops and livestock species while biotechnology provides the means of conserving and strengthening biodiversity. Most plant and animal species from the earlier ages have lost some ecologically important traits such as resistance to pests and pathogens, resistance to harsh environmental conditions, and adaptation to various soil and climate conditions. From an economical standpoint, standards such as increased milk and meat production of domestic and farm animals as well as environmental conditions are being viewed as very important, which is why the use of biotechnology is drawing a large interest with its tremendous potential for efficient, ecofriendly, and economically viable options for conserving biodiversity.

Biotechnology and biodiversity depend on one another as biotechnology depends on the availability of natural resource due to biodiversity, and biodiversity depends on the management of biotechnology. Along with increasing productivity and eliminating the use of chemicals, biotechnology also enhances natural agrobiological systems. It has become a preferable tool to adding conserving and strengthening biodiversity, and recent advances in genomic, proteomic, and metabolomics research opens up many opportunities in the different fields associated with biotechnology [1].

\section{RELATIONSHIP TO PUBLIC HEALTH}

Both birds and humans are involved in the public health concern, therefore it is important to look at both animal health and human health. Veterinarians, farmers, and stakeholders are all involved with animal health issues, and they are necessary to help minimize the threat of H5N1 in humans through the maintenance of the poultry by methods such as monitoring and controlling disease outbreak, improving bio-security in poultry production and trade, and strengthening surveillance measures. In regards to human health, the Department of Health is essential for controlling disease outbreaks through monitoring the disease and evaluating disease management. It is also critical to involve physicians from the private sector and health professionals to monitor disease outbreaks in the human population [15].

Implementing buildings dedicated to disease management should be a focus for reducing and ultimately eliminating avian influenza. Training individuals that handle poultry marketing or work with health care will be needed. Teaching specific techniques, providing supplies for training improvement, and compensation following culling operations are crucial throughout the development of these projects [15].

In addition to facilities and training sessions dedicated for those involved in disease management and prevention of an avian influenza outbreak, raising awareness through campaigns and the help of the media is important. The campaigns would address the sanitation and hygiene practices regarding avian 
influenza, as well as the risk factors involved with the spread of avian influenza. Along with raising awareness, regulations and standards regarding sanitation and hygiene must be issued. The final installment for control and prevention of an avian influenza outbreak is the use of vaccines [16]. As mentioned in the previous section, modern biotechnology advancements have led to DNA marking techniques, which have developed cheaper, safer, and more effective vaccines to prevent viral infections.

\section{CONCLUSIONS}

In the literature review section, we looked at how avian influenza emerges among birds, how flocks of birds transmit the virus among other flocks and species through migration patterns, avian influenza in chickens in Bangladesh, the biodiversity of certain regions in Bangladesh, and the impacts of biodiversity on the emergence and transmission of infectious diseases. Also, from the research efforts through biotechnology, we discussed different methods of biotechnology among animals, the role of biotechnology in today's world, and how biotechnology can be applied to conserve biodiversity. Based on the information discussed in the previous sections, we will now come up with conclusions to the questions posed in the introduction.

As mentioned earlier, the loss of biodiversity tends to increase pathogen transmission and disease incidence. There were a couple of cases mentioned where the opposite is true, but in this case, the hosts vary (different species of birds) and the ecosystems vary (different regions of the world are effected by AI). Going back to the example involving a different virus, the West Nile virus, it is clear that communities in the United States with low avian diversity are dominated by the species that spread the virus, resulting in a high volume of infected mosquitoes and humans.

With this in mind, increasing the biodiversity with the help of biotechnology will reduce the transmission and burden of avian influenza in Bangladesh. The use of vaccines containing purified natural proteins made from DNA marker technology is a realistic solution to the avian influenza issue in Bangladesh.

\section{RECOMMENDATIONS}

From the answers and conclusions given in the previous section, we have a strong idea of what should be done to solve the problem we are looking at. Now we have to apply those ideas, which can be done in a few ways.

Research in animal biotechnology can lead to saving species of birds (domestic, wild, or migratory) that are endangered or near extinction. In turn, this will lead to a greater biodiversity, which will ultimately lead to reducing (even eliminating) the transmission of avian influenza between different species in Bangladesh. The ultimate result of this process will be healthy poultry that will help feed the large, growing population of Bangladesh, as well as economic and social benefits to farmers and many others.

DNA recombinant technology has enormous potential, and modern advances in biotechnology has led to the development of subunit vaccines that are cheaper, safer, and more effective against different diseases than traditional vaccines. Utilizing this technology for poultry can yield promising results, leading to an increase in biodiversity, and ultimately reducing the burden of the avian influenza in Bangladesh.

Two areas of biotechnology that are suitable for collaboration in developing countries are medicinal (red) biotechnology and environmental (green) biotechnology. Medicinal biotechnology has been used to produce hundreds of different medicines over the past few decades by genetically modifyingcertain gene expressions and amino acids. Also, as mentioned earlier in the paper, vaccines produced from DNA recombinant technology has produced safer, cheaper, and more effective results against different diseases.Medicinal biotechnology is the main area that is suitable for collaboration. Environmental biotechnology is used in waste treatment and preventing pollution. Since the ecosystem and environment are essential for biodiversity, environmental biotechnology can be used to improve the biodiversity of certain areas. It is an interesting area of research, but medicinal biotechnology dealing with animal biotechnology should be the area for collaboration among developing countries.

Funding for research efforts can come from a variety of sources, but government grants and research through universities are the most suitable candidates. Also, training facilities need to be developed to train for disease management and prevention of avian influenza outbreaks. Along with training facilities, management centers and campaigns raising awareness on how to prevent the infection and spread of avian influenza must come along. Media involvement will help raise awareness of avian influenza, especially on the prevention of transmission and acquirement of the virus. Farmers ranging from backyard farmers to large farming grounds must become informed about proper vaccination treatments. 


\section{REFERENCES}

[1] Roberton SI., Bell DJ., Smith GJD., Nicholls M., Chan KH., Nguyen DR., Preiris JSM., “Avian influenza H5N1 in viverrids: implications for wildlife healthand conservation”, Proc. R. Soc. B, vol. 273, pp. 1729-1732, 2006. doi:10.1098/rspb.2006.3549.

[2] Olsen B., Munster VJ., Wallensten A., Waldenström J., Osterhaus ADME., Fouchier RAM., “Global Patterns of Influenza A Virus in Wild Birds”, Science Magazine, vol. 312, pp.384-388, 2006.

[3] Swayne DE., Spackman E., Pantin-Jackwood M., "Success Factors for Avian Influenza Vaccine Use in Poultry and Potential Impact at the Wild Bird-Agricultural Interface”, EcoHealth, vol. 11, pp. 94-108, 2014.

[4] Boyce WM., Sandrock C., Kreuder-Johnson C., Kelly T., Cardona C., “Avian influenza viruses in wild birds: A moving target”, Science Direct, vol. 32, pp. 275-286, 2009.

[5] Beaudoin AL., Kitikoon P., Schreiner PJ., Singer RS., Sasipreeyajan J., Amonsin A., Bender JB., "Risk Factors for Exposure to Influenza A Viruses, Including Subtype H5 Viruses, in Thai Free-Grazing Ducks”, Transboundary and Emerging Diseases, vol. 61, pp. 362-374, 2014.

[6] Keesing F., Belden LK., Daszak P., Dobson A., Harvell CD., Holt RD., Ostfeld RS., "Impacts of biodiversity on the emergence and transmission of infectious diseases”, Nature, vol. 468, pp. 647-652, 2010.

[7] Biswas PK., Christensen JP., Ahmed SSU., Barua H., Das A., Rahman MH., Debnath NC., "Avian Influenza Outbreaks in Chickens, Bangladesh”, Centers for Disease Control and Prevention, vol. 14, pp. 1909-1912, 2008.

[8] Islam MJ., Anisuzzaman M., Sarker MJ., Minar MH., Majumdar MZ., Islam T., "Floral and Bird Biodiversity of DingaputaHaor and Its Surrounding Area of MohangongUpazila, Netrakona District”, World Journal of Agriculture Research, vol. 4, pp. 70-76, 2013.

[9] Benson EE., "Plant Conservation Biotechnology”, Philadelphia, PA: Taylor \& Francis Inc, 2007.

[10] Swayne DE., Pantin-Jackwood M., Kapczynski D., Spackman E., Suarez DL., "Susceptibility of Poultry to Pandemic (H1N1) 2009 Virus”, Center for Disease Control and Prevention, vol. 15, pp. 2061-2063, 2009.

[11] Babiuk LA., Phillips JP., “Animal Biotechnology: Comprehensive Biotechnology”, First Supplement. Elmsford, NY: Pergamon Press, 1989.

[12] Green Peace, "Smart Breeding Marker-Assisted Selection: A non-invasive biotechnology alternative to genetic engineering of plant varieties”. Retrieved from http://www.greenpeace.org/australia/PageFiles/348427/smartbreeding.pdf, 2009.

[13] Artificial Insemination, "Encyclopaedia $\quad$ Britannica”, Retrieved from http://www.britannica.com/EBchecked/topic/37134/artificial-insemination, 2014.

[14] Flanders F., “Exploring Animal Science”, Clifton Park, NY: Cengage Learning, 2011.

[15] Haider M., Applebaum B., "Disease Management of Avian Influenza H5N1 in Bangladesh - A Focus on Maintaining Healthy Live Birds", Health Management - Different Approaches and Solutions, Dr. Krzysztof Smigorski (Ed.), ISBN: 978-953-307-296-8, InTech, DOI: 10.5772/20443, 2011.

[16] Haider M., Frank J., “Avian and Pandemic Influenza (API):A Prevention-Oriented Approach, Molecular Virology”, Mr. Moses Adoga (Ed.), ISBN: 978-953-51-0369-1, InTech, DOI: 10.5772/39237, 2012. 